\title{
COADJOINT EQUIVARIANCY OF MOMENTUM MAPPING
}

\author{
By Kentaro Mikami
}

1. A coadjoint orbit of a Lie group $G$ is a $G$-homogeneous symplectic manifold with the inclusion map as the coadjoint equivariant momentum mapping. If $(M, \Omega)$ is a $G$-homogeneous symplectic manifold with the coadjoint equivariant momentum mapping, then the coadjoint equivariant momentum mapping gives a symplectic covering mapping onto a coadjoint orbit. And we have a result of B. Kostant which classifies the (simply connected) homogeneous symplectic manifolds with coadjoint equivariant momentum mappings (cf. [1], [2], [5]). An action with a fixed point may be considered to be antipodal with a homogeneous action. As explained in [4], we have some theorems concerning with the existence of (coadjoint equivariant) momentum mappings. In particular, the following three guarantee the coadjoint equivariancy of a momentum mapping:

(1) $H^{2}(\mathfrak{g}, R)=0$, where $\mathrm{g}$ is the Lie algebra of $G$ (cf. [4], [5], [6]),

(2) the symplectic form is an exact form of a $G$-invariant 1-form (cf. [1]), and

(3) $G$ is a semidirect product of $G_{1}$ by $G_{2}$, where $G_{1}$ and $G_{2}$ have coadjoint equivariant momentum mappings, $H^{1}\left(\mathfrak{g}_{1}, R\right)=0$ and $G_{1}$ is connected (cf. [3], [4]).

In this paper, we give a condition for the coadjoint equivariancy of momentum mappings. The result is

Proposition. Let $(M, \Omega)$ be a connected symplectıc manifold, and let $G$ be a symplectic action on $(M, \Omega)$ with a momentum mapping. If the action $G$ has a fixed point, then $G$ has a coadjoint equivariant momentum mapping.

2. Let $(M, \Omega)$ be a connected symplectic manifold, that is, $M$ is a connected smooth manifold with a non-degenerate closed 2-form $\Omega$. $\Omega$ induces a bundle isomorphism $\Omega^{\mathrm{b}}: T M \rightarrow T^{*} M$ between the tangent bundle and the cotangent bundle of $M$ defined by

$$
\left.\Omega^{\mathrm{b}}(v)=v\right\lrcorner \Omega .
$$

Denote the inverse of $\Omega^{b}$ by $\Omega^{\#}$. $\Omega^{\#}: T^{*} M \rightarrow T M$ is also a bundle isomorphism. Let $C^{\infty}(M)$ (resp. aut $(M, \Omega)$ ) be the set of all real valued smooth functions (resp. Hamiltonian vector fields i. e., vector field $X$ satisfying $L_{X} \Omega=0$ ) on $M$.

For each $f \in C^{\infty}(M)$, define $\beta(f)$ by

Received August 24, 1982 


$$
\beta(f)=\Omega^{\#}(d f) \text {, or equivalently, } \Omega^{b}(\beta(f))=d f \text {. }
$$

Since we have

$$
\begin{aligned}
L_{\beta(f)} \Omega & =\beta(f)\lrcorner d \Omega+d(\beta(f)\lrcorner \Omega) \\
& =d\left(\Omega^{\mathrm{b}}(\beta(f))\right)=d(d f)=0,
\end{aligned}
$$

$\beta(f) \in \operatorname{aut}(M, \Omega)$ for each $f \in C^{\infty}(M)$. For each $f, h$ in $C^{\infty}(M)$, the Poisson bracket $\{f, h\}$ is defined by

$$
\begin{aligned}
\{f, h\} & =-\Omega(\beta(f), \beta(h)) \\
& =\beta(f)\lrcorner d h .
\end{aligned}
$$

$C^{\infty}(M)$ is a Lie algebra with the Poisson bracket $\{$,$\} . For example, the Jacobi$ identity for $\{$,$\} comes from d \Omega=0$. It is clear that $\operatorname{aut}(M, \Omega)$ is a Lie subalgebra of the Lie algebra of all smooth vector fields on $M$. Since we have

$$
\begin{aligned}
d\{f, h\} & =d(\beta(f)\rfloor d h) \\
& =L_{\beta(f)} d h \\
& \left.=L_{\beta(f)}(\beta(h)\lrcorner \Omega\right) \\
& =[\beta(f), \beta(h)]\lrcorner \Omega \\
& =\Omega^{b}[\beta(f), \beta(h)],
\end{aligned}
$$

$\beta$ satisfies $\beta\{f, h\}=[\beta(f), \beta(h)]$, that is, $\beta$ is a Lie algebra homomorphism from $C^{\infty}(M)$ into aut $(M, \Omega)$.

Since $\left.L_{X} \Omega=d X\right\lrcorner \Omega=d\left(\Omega^{\mathrm{b}}(X)\right)$, for each Hamiltonian vector field $X$ we have a closed 1-form $\Omega^{\mathrm{b}}(X)$. Let $\gamma(X)$ be the de Rham cohomology class of $\Omega^{\mathrm{b}}(X)$ for each $X$ in $\operatorname{aut}(M, \Omega)$. We have

$$
\begin{aligned}
\Omega^{\mathrm{b}}([X, Y]) & =[X, Y]\lrcorner \Omega \\
& \left.=L_{X}(Y\lrcorner \Omega\right) \\
& =d(X\lrcorner Y\lrcorner \Omega) \quad \text { for each } X, Y \text { in } \operatorname{aut}(M, \Omega) .
\end{aligned}
$$

This implies $\gamma[X, Y]=0$. Thus $\gamma$ is a Lie algebra homomorphism from $\operatorname{aut}(M, \Omega)$ into $H^{1}(M, \boldsymbol{R})$ if we introduce the trivial Lie algebra structure in $H^{1}(M, \boldsymbol{R})$. We have the following short exact sequence of Lie algebra homomorphisms

$$
0 \longrightarrow R \subset C^{\infty}(M) \stackrel{\beta}{\longrightarrow} \operatorname{aut}(M, \Omega) \stackrel{\gamma}{\longrightarrow} H^{1}(M, \boldsymbol{R}) \longrightarrow 0 .
$$

Let $G$ be a Lie group. A smooth left action $\phi: G \times M \rightarrow M$ is called a symplectic action of a Lie group $G$ on a symplectic manifold $(M, \Omega)$ if for each $g$ in $G$ the map $\phi_{g}: M \rightarrow M: m \mapsto \phi(g, m)$ is a symplectomorphism, that is, $\phi_{g}^{*} \Omega$ $=\Omega$. A symplectic action $(G, \phi)$ on $(M, \Omega)$ induces a Lie algebra homomorphism 
defined by

$$
\rho: g \longrightarrow \operatorname{aut}(M, \Omega)
$$

$$
\rho(\xi)_{m}=\left(\psi_{m}\right)_{* e}(-\xi),
$$

where $g$ is the Lie algebra of $G$ consisting of all left-invariant vector fields on $G, \psi_{m}$ is the orbit map of $(G, \phi)$ through $m$, that is, $\phi_{m}(g)=\phi(g, m)$ and $e$ is the unit of $G$.

A momentum mapping for the symplectic action $(G, \phi)$ is a mapping $J: M$ $\rightarrow \mathrm{g}^{*}$ (=the dual space of $\mathrm{g}$ ) such that

$$
d\langle J, \xi\rangle=\rho(\xi)\lrcorner \Omega .
$$

This condition is equivalent to

or

$$
d \hat{J}(\xi)=\Omega^{b}(\rho(\xi))
$$

$$
\Omega^{\#} d \hat{J}(\xi)=\rho(\xi) \quad \text { i. e., } \quad \beta(\hat{J}(\xi))=\rho(\xi),
$$

where $\hat{J}(\xi)$ in $C^{\infty}(M)$ is defined by

$$
\hat{J}(\xi)(m)=\langle J(m), \xi\rangle .
$$

A momentum mapping $J: M \rightarrow \mathrm{g}^{*}$ for the symplectic action $(G, \phi)$ is coadjoint equivariant if and only if $J$ satisfies

$$
J\left(\phi_{g}(m)\right)=A d\left(g^{-1}\right)^{*} J(m)
$$

for each $g, m$ in $G$ and $M$. This condition is equivalent to

$$
\hat{J} A d\left(g^{-1}\right)=\phi_{g}^{*} \hat{J} \quad \text { for each } g \text { in } G .
$$

Differentiating the above, we have

$$
\hat{J}[\xi, \eta]=\{\hat{J}(\xi), \hat{J}(\eta)\} \quad \text { for each } \xi, \eta \text { in } g,
$$

that is, $\hat{J}$ is a Lie algebra homomorphism of $g$ into $C^{\infty}(M)$. We know that if the Lie group $G$ is connected and if $\hat{J}$ is a Lie algebra homomorphism, then $J$ is coadjoint equivariant.

\section{At first, we prove}

LemMA (cf. [1]). For a symplectıc action $(G, \phi)$ on a symplectic manifold $(M, \Omega)$ we have
(1) $\phi_{g}^{*} \Omega^{b} \phi_{g *}=\Omega^{b}$
(2) $\phi_{g *} \Omega^{\#} \phi_{g}^{*}=\Omega^{\#}$
(3) $\phi_{g *} \beta(f)=\beta\left(\phi_{g-1}^{*} f\right)$
(4) $\phi_{g}^{*}\{f, h\}=\left\{\phi_{g}^{*} f, \phi_{g}^{*} h\right\}$
(5) $\phi_{g *} \rho(\xi)=\rho(A d(g) \xi)$ 
for each $g$ in $G, f, h$ in $C^{\infty}(M)$ and $\xi$ in $g$. These mean that the diagrams below are commutative.
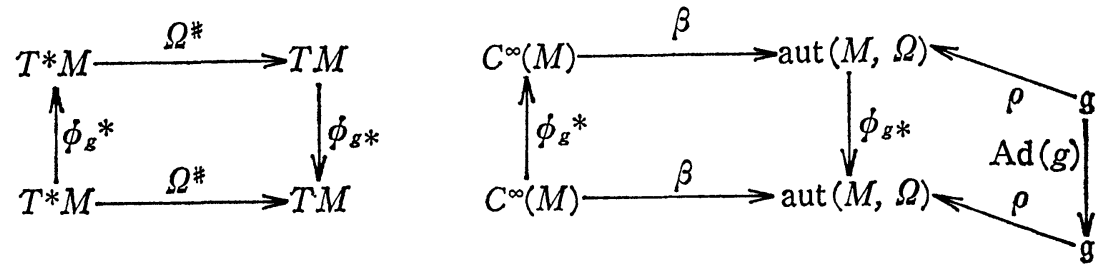

Proof of Lemma. For each $g$ in $G, \phi_{g}^{*} \Omega=\Omega$ implies

$$
\Omega\left(\phi_{g *} v, \phi_{g *} w\right)=\Omega(v, w) \quad \text { for any } v, w \text { in } T_{m} M .
$$

This means

$$
\left(\phi_{g}^{*} \Omega^{b}\left(\phi_{g * v}\right)\right)(w)=\left(\Omega^{b}(v)\right)(w)
$$

and proves (1). (2) is only another expression of (1). (2) implies (3), since

$$
\begin{aligned}
\phi_{g *} \beta(f) & =\phi_{g *} \Omega^{\#}(d f)=\Omega \#\left(\phi_{g-1}^{*} d f\right)=\Omega \#\left(d \phi_{g-1}^{*} f\right) \\
& =\beta\left(\phi_{g-1}^{*} f\right) .
\end{aligned}
$$

The proof of (4) is

$$
\begin{aligned}
\phi_{g}^{*}\{f, h\} & \left.\left.=\phi_{g}^{*}(\beta(f)\lrcorner d h\right)=\left(\phi_{g-1} * \beta(f)\right)\right\lrcorner \phi_{g}^{*} d h \\
& \left.=\beta\left(\phi_{g}^{*} f\right)\right\lrcorner d\left(\phi_{g}^{*} h\right)=\left\{\phi_{g}^{*} f, \phi_{g}^{*} h\right\}
\end{aligned}
$$

using (3). (5) comes from

$$
\begin{aligned}
\phi_{g *}\left(\rho(\xi)_{m}\right) & =\phi_{g *}\left(\phi_{m}\right)_{* e}(-\xi)=\left(\phi_{g} \psi_{m}\right)_{* e}(-\xi) \\
& =\left(\phi_{\dot{\phi}(g, m)} I_{g}\right)_{* e}(-\xi)=\left(\phi_{\phi(g, m)}\right)_{* e}(-A d(g) \xi) \\
& =\rho(A d(g) \xi)_{\phi(g, m)},
\end{aligned}
$$

where $I_{g}$ is the inner automorphism of $G$ induced by $g$ and $\left(I_{g}\right)_{* e}=A d(g)$.

Now we prove our Proposition. Let $J$ be a given momentum mapping for the symplectic action $(G, \phi)$, that is, $\hat{J}$ is linear and satisfies $\beta \hat{J}=\rho$. Using Lemma, we have

Therefore

$$
\begin{aligned}
\beta\left(\phi_{g}^{*} \hat{J}(\xi)\right) & =\phi_{g^{-1}}^{*} \beta \hat{J}(\xi)=\phi_{g-1}^{*} \rho(\xi)=\rho\left(A d\left(g^{-1}\right) \xi\right) \\
& =\beta \hat{J}\left(A d\left(g^{-1}\right) \xi\right) \quad \text { for each } g \text { in } G .
\end{aligned}
$$

$$
\phi_{g}^{*} \hat{J}(\xi)-\hat{J}\left(A d\left(g^{-1}\right) \xi\right)
$$

is a constant function on $M$, and so 


$$
\phi_{g}^{*} \hat{J}(\xi)-\hat{J}\left(A d\left(g^{-1}\right) \xi\right)=\left(\phi_{g}^{*} \hat{J}(\xi)\right)\left(m_{0}\right)-\hat{J}\left(A d\left(g^{-1}\right) \xi\right)\left(m_{0}\right)
$$

for any given point $m_{0}$ in $M$. Let $m_{0}$ be a fixed point for the symplectic action $(G, \phi)$, that is, $\phi_{g}\left(m_{0}\right)=m_{0}$ for each $g$ in $G$. Then we have

$$
\phi_{g}^{*} \hat{J}(\xi)-\hat{J}\left(A d\left(g^{-1}\right) \xi\right)=\hat{J}(\xi)\left(m_{0}\right)-\hat{J}\left(A d\left(g^{-1}\right) \xi\right)\left(m_{0}\right)
$$

for each $g$ in $G$. Define $\lambda: g \rightarrow C^{\infty}(M)$ by

$$
\lambda(\xi)=\hat{J}(\xi)-\hat{J}(\xi)\left(m_{0}\right) .
$$

Then $\lambda$ is linear and satisfies $\beta \lambda=\rho$. Moreover we have

$$
\begin{aligned}
\phi_{g}^{*} \lambda(\xi) & =\phi_{g}^{*} \hat{J}(\xi)-\hat{J}(\xi)\left(m_{0}\right) \\
& =\hat{J}\left(A d\left(g^{-1}\right) \xi\right)-\hat{J}\left(A d\left(g^{-1}\right) \xi\right)\left(m_{0}\right) \\
& =\lambda\left(A d\left(g^{-1}\right) \xi\right) .
\end{aligned}
$$

Thus $\lambda$ defines a coadjoint equivariant momentum mapping for the symplectic action $(G, \phi)$. This completes the proof of our Proposition.

Acknowledgement. The author would like to express his sincere thanks to Professors Y. Hatakeyama, S. Tanno, H. Kitahara, T. Takahashi and S. Yorozu for their encouragement and helpful suggestions.

\section{REFERENCES}

[1] R. Abraham and J.E. Marsden, Foundations of mechanics, Benjamin, New York, 1978.

[2] B. Kostant, Quantizations and Unitary representations, Lecture Notes in Math., vol. 170, Springer-Verlag, Berlin, 1970, pp. 87-208.

[3] K. Miкамi, The existence of a coadjoint equivariant momentum mapping for a semidirect product, Proc. Amer. Math. Soc., vol. 82, 1981, pp. 465-469.

[4] K. Mikami, Another proof of existence of momentum mappings, Kodai Math. J., 6 (1983), pp. 198-203.

[5] N.R. Wallach, Symplectic geometry and Fourier analysis, Math. Sci. Press, 1970.

[6] A. Weinstein, Lectures on symplectic manifolds, CBMS Regional Conf. Ser. in Math., no. 29, Amer. Math. Soc., 1977. 